\title{
Gain-induced switching in metal-dielectric-metal plasmonic waveguides
}

\author{
Zongfu Yu ${ }^{\mathrm{a}^{*}}$, Georgios Veronis ${ }^{\mathrm{a}}$, Mark L. Brongersma ${ }^{\mathrm{b}}$, Shanhui Fan ${ }^{\mathrm{a}}$ \\ ${ }^{\mathrm{a}}$ Ginzton Laboratory, Stanford University, Stanford, California, U.S.A. 94305; \\ ${ }^{\mathrm{b}}$ Geballe Laboratory of Advanced Materials, Stanford University, Stanford, California, U.S.A. 94305
}

\begin{abstract}
The authors show that the incorporation of gain media in only a selected device area can annul the effect of material loss, and enhance the performance of loss-limited plasmonic devices. In addition, they demonstrate that optical gain provides a mechanism for on/off switching in metal-dielectric-metal (MDM) plasmonic waveguides. The proposed gain-assisted plasmonic switch consists of a subwavelength MDM plasmonic waveguide side-coupled to a cavity filled with semiconductor material. In the absence of optical gain in the semiconductor material filling the cavity, an incident optical wave in the plasmonic waveguide remains essentially undisturbed by the presence of the cavity. Thus, there is almost complete transmission of the incident optical wave through the plasmonic waveguide. In contrast, in the presence of optical gain in the semiconductor material filling the cavity, the incident optical wave is completely reflected. They show that the principle of operation of such gain-assisted plasmonic devices can be explained using a temporal coupled-mode theory. They also show that the required gain coefficients are within the limits of currently available semiconductor-based optical gain media.
\end{abstract}

Keywords: Plasmonic waveguides, switch, metal-dilectric-metal waveguide, optical gaining, coupled modes

\section{INTRODUCTION}

Plasmonic devices, based on surface plasmons propagating at metal-dielectric interfaces, have shown the potential to guide and manipulate light at deep subwavelength scales [1]. In plasmonic waveguides, the propagation length of the supported optical modes is limited by the material loss in the metal. The use of gain media has been suggested as a means to compensate for the material loss, or to amplify surface plasmons $[2,3]$. Such use of gain media has been demonstrated experimentally $[4,5]$. It has also been suggested that the incorporation of media with realistic gain coefficients in plasmonic devices can lead to low-attenuation or even lossless propagation of surface plasmons in plasmonic waveguides[6-8], increase of the resolution of negative-refractive-index near-field lenses [9], and control of the group velocity of nanoscale plasmonic waveguides [10].

In this letter, we introduce a different use of gain in plasmonic devices. We show that the incorporation of gain media in only a selected device area can annul the effect of material loss, and enhance the performance of loss-limited plasmonic devices. In addition, we demonstrate that optical gain provides a mechanism for on/off switching in metal-dielectric-metal (MDM) plasmonic waveguides. The proposed gain-assisted plasmonic switch consists of a subwavelength MDM plasmonic waveguide side-coupled to a cavity filled with semiconductor material. In the absence of optical gain in the semiconductor material filling the cavity, an incident optical wave in the plasmonic waveguide remains essentially undisturbed by the presence of the cavity. Thus, there is almost complete transmission of the incident optical wave through the plasmonic waveguide. In contrast, in the presence of optical gain in the semiconductor material filling the cavity, the incident optical wave is completely reflected. We show that the principle of operation of such gain-assisted plasmonic devices can be explained using a temporal coupled-mode theory. We also show that the required gain coefficients are within the limits of currently available semiconductor-based optical gain media.

\section{DEVICE STRUCTURE AND SIMULATION}

We consider a subwavelength gold-air-gold MDM plasmonic waveguide side-coupled to a rectangular cavity [11, 12]

Integrated Optics: Devices, Materials, and Technologies XII, edited by Christoph M. Greiner, Christoph A. Waechter Proc. of SPIE Vol. 6896, 68960L, (2008) · 0277-786X/08/\$18 - doi: 10.1117/12.764052 
filled with semiconductor material with dielectric permittivity $\varepsilon=\varepsilon_{r}+i \varepsilon_{i}$. [inset of Fig. 1(d)]. We use a two-dimensional finite-difference frequency-domain (FDFD) method $[13,14]$ to calculate the transmission $T$ and reflection $R$ coefficients of such a structure. This method allows us to directly use experimental data for the frequency-dependent dielectric constant of metals such as gold [15], including both the real and imaginary parts, with no approximation. Perfectly matched layer (PML) absorbing boundary conditions are used at all boundaries of the simulation domain [16].
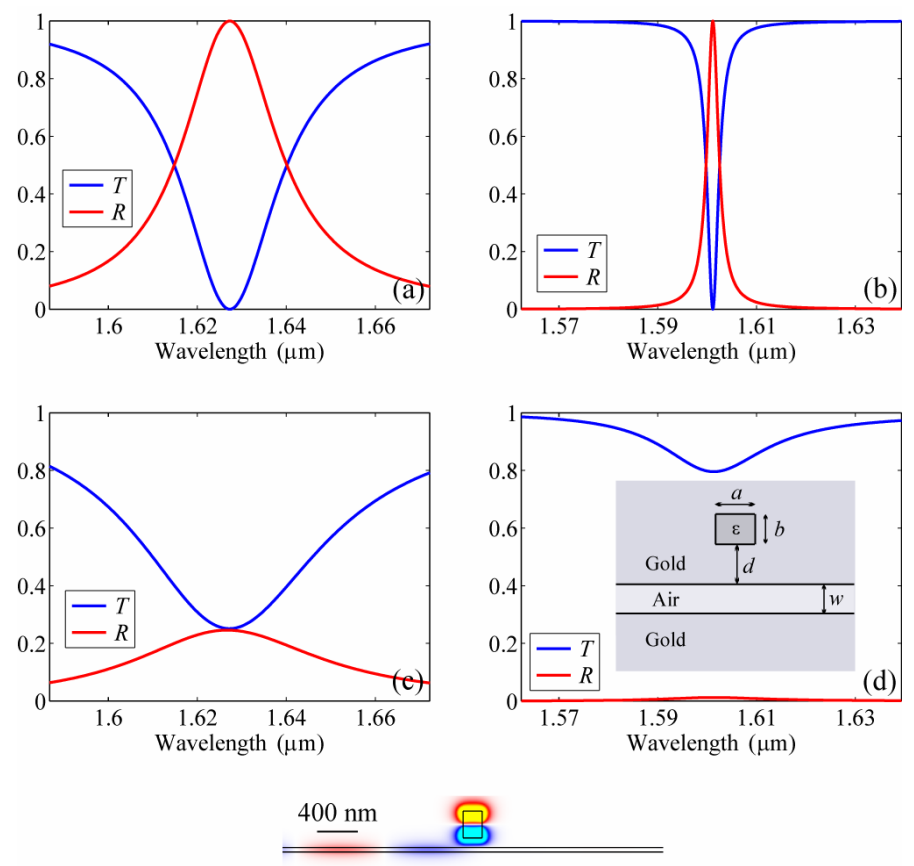

(e)

Fig. 1. (a) Transmission $T$ and reflection $R$ spectra of a gold-air-gold MDM plasmonic waveguide side-coupled to a rectangular cavity [shown in the inset of Fig. 1(d)] calculated using FDFD. Results are shown for $w=50 \mathrm{~nm}, d=50$ $\mathrm{nm}, a=200 \mathrm{~nm}$, and $b=280 \mathrm{~nm}$. The cavity is filled with silicon ( $\varepsilon=12.25$ ), and the metal is assumed to be lossless $\left(\varepsilon_{\mathrm{m} e t}=\varepsilon_{\text {met, real }}\right.$, neglecting the imaginary part of the dielectric permittivity $\varepsilon_{\text {met,imag }}$ ). (b) Same as Fig. 1(a), except $d=100 \mathrm{~nm}$. (c) Same as Fig. 1(a), except that the material loss in the metal is included. (d) Same as Fig. 1(b), except that the material loss in the metal is included. (e) Magnetic field profile of the device at resonance ( $\lambda=1.6012 \mu \mathrm{m}$ ). All other parameters are as in Fig. 1(b).

A system consisting of a waveguide side-coupled to a cavity, which supports a resonant mode of frequency $\omega_{0}$, can be described analytically. Using coupled-mode theory [17], it can be shown that the transmission $T$ and reflection $R$ coefficients of the system are given by

$$
\begin{aligned}
& T=\frac{\left(\omega-\omega_{0}\right)^{2}+\left(1 / \tau_{0}\right)^{2}}{\left(\omega-\omega_{0}\right)^{2}+\left(1 / \tau_{0}+1 / \tau_{e}\right)^{2}} \\
& R=\frac{\left(1 / \tau_{e}\right)^{2}}{\left(\omega-\omega_{0}\right)^{2}+\left(1 / \tau_{0}+1 / \tau_{e}\right)^{2}}
\end{aligned}
$$

where $\omega$ is the frequency, $1 / \tau_{e}$ is the decay rate of the field in the cavity due to the power escape through the waveguide, and $1 / \tau_{0}$ is the decay rate due to the internal loss in the cavity. We observe that far from the resonant frequency $\omega_{0}$, the 
cavity mode is not excited, and the incident waveguide mode is completely transmitted. At resonance, if there is no internal loss in the cavity $\left(1 / \tau_{0}=0\right)$, the incident mode is completely reflected, and the spectral width of the resonance is determined solely by the strength of the coupling between the waveguide and the cavity $\left(1 / \tau_{e}\right)$.

We first assume that the metal is lossless (i.e. neglecting the imaginary part $\operatorname{Im}\left[\varepsilon_{\text {metal }}\right]$ of metal dielectric permittivity $\varepsilon_{\text {metal }}$ ). In Figs. 1(a) and 1(b) we show the transmission $T$ and reflection $R$ coefficients of the device [inset of Fig. 1(d)] as a function of the wavelength $\lambda$ for $d=50 \mathrm{~nm}, d=100 \mathrm{~nm}$, respectively, calculated using FDFD ( $d$ is the spacing between the cavity and the waveguide). The dimensions of the cavity $a$ and $b$ [inset of Fig. 1(d)], which is filled with silicon ( $\varepsilon=12.25$ ), are chosen so that its resonant frequency is in the near-infrared wavelength range. Since the metal is assumed to be lossless, there is no internal loss in the cavity, and there is therefore complete reflection at resonance, as predicted from coupled-mode theory. In addition, in the side-coupled structure the coupling strength can be controlled by the distance $d$ [inset of Fig. 1(d)]. Increased the distance $d$ between the waveguide and the cavity results in a weaker coupling, and therefore higher quality factor $Q$ and narrower spectral width of the resonance. We also observe that the resonant frequency of the cavity $\omega_{0}$ slightly shifts when $d$ is varied.

We next consider the effect of the material loss in the metal on the performance of the device. In Figs. 1(c) and 1(d) we show the transmission $T$ and reflection $R$ spectra of the device [inset of Fig. 1(d)] for $d=50 \mathrm{~nm}, d=100 \mathrm{~nm}$, respectively, when the material loss in the metal is included. We observe that the on-resonance transmission increases, and the on-resonance reflection decreases with respect to the lossless case, when the effect of loss in the metal is taken into account. We also observe that the on-resonance transmission increases and the on-resonance reflection decreases as the waveguide-cavity coupling becomes weaker $\left(1 / \tau_{e}\right.$ decreases). Both of these effects are consistent with coupled-mode theory (Eqs. (1) and (2)).
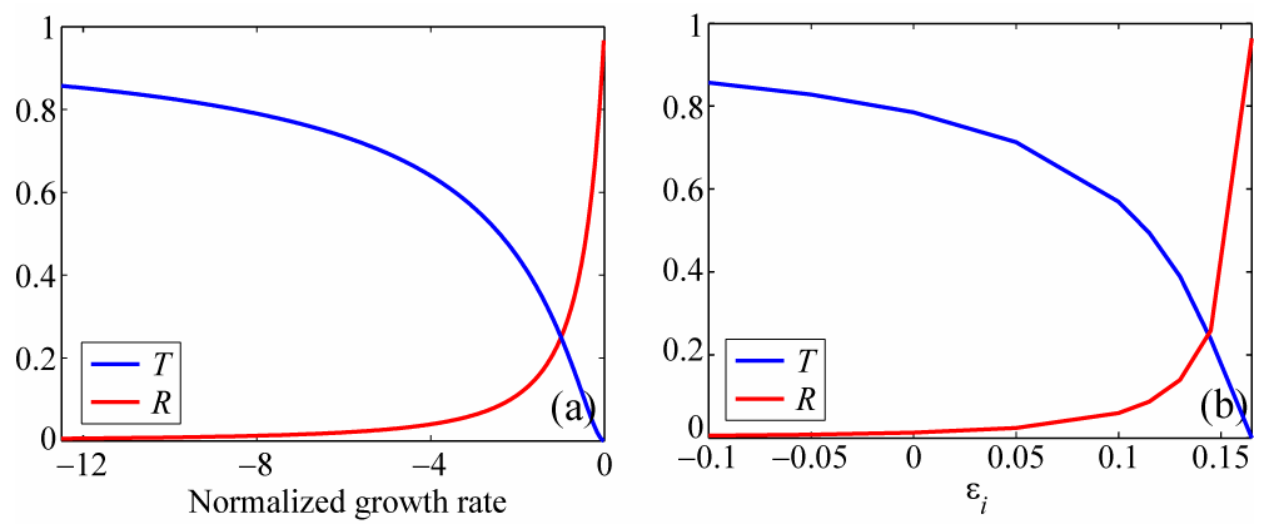

Fig. 2. (a) On-resonance transmission $T$ and reflection $R$ coefficients of a waveguide side-coupled to a cavity as a function of the normalized growth rate $\left(1 / \tau_{g}-1 / \tau_{0}\right) /\left(1 / \tau_{e}\right)$ calculated using coupled-mode theory. (b) Transmission $T$ and reflection $R$ coefficients of the device [shown in the inset of Fig. 1(d)] as a function of $\varepsilon_{i}$ at $\lambda=1.5452 \mu$ m calculated using FDFD. The cavity is filled with $\operatorname{InGaAsP}\left(\varepsilon=11.38+i \varepsilon_{i}\right)$. All other parameters are as in Fig. 1(d).

We now consider the effect of incorporating gain in the semiconductor material filling the cavity. Theoretically, the effect of optical gain can be included in Eqs. (1) and (2) by replacing $1 / \tau_{0}$ is with $1 / \tau_{0}-1 / \tau_{g}$, where $1 / \tau_{g}$ is the growth rate of the field in the cavity due to the gain of the material. In Fig. 2(a) we show the on-resonance transmission and reflection coefficients of the device as a function of the normalized growth rate $\left(1 / \tau_{g}-1 / \tau_{0}\right) /\left(1 / \tau_{e}\right)$ calculated using 
coupled-mode theory. We observe that when $1 / \tau_{g}=1 / \tau_{0}$, the gain does not have much effect, and there is very low reflection and high transmission at resonance. On the other hand, when $1 / \tau_{g}=1 / \tau_{0}$, the decay of the cavity field due to the material loss in the metal is compensated by the gain. In such a case coupled-mode theory predicts complete reflection and no transmission at resonance. In other words, the device behaves essentially as if the metal were lossless [Figs. 1(a) and 1(b)]. Note also that in this regime the device does not lase.

As a concrete numerical example of the concept presented above, we fill the cavity of the same structure as in the inset of Fig. 1(d) with a InGaAsP gain medium $\left(\varepsilon=11.38+i \varepsilon_{i}\right)$. In the absence of pumping such a medium is lossy and $\varepsilon_{i}$ is negative. With pumping, $\varepsilon_{i}$ increases with pump power and eventually becomes positive when the medium exhibits gain. In Fig. 2(b) we show the transmission $T$ and reflection $R$ coefficients of the device as a function of $\varepsilon_{i}$ at $\lambda=1.5452 \mu \mathrm{m}$, which is the resonant wavelength for $\varepsilon_{i}=0.165$ as calculated using FDFD. We observe that there is excellent agreement with the results of the coupled-mode theory for the on-resonance transmission and reflection. This is due to the fact that the resonant frequency of the cavity has only a weak dependence on $\varepsilon_{i}$, since $\varepsilon_{i}=\varepsilon_{r}=11.38$. Complete reflection is obtained for $\varepsilon_{i} ; 0.165$ which corresponds to a gain coefficient of $g ; 2 \times 10^{3} \mathrm{~cm}^{-1}$. Such a gain coefficient is within the limits of currently available semiconductor gain media[6, 18, 19]. In Figs. 3(b) and 3(d) we show the transmission $T$ and reflection $R$ spectra, and the magnetic field profile at resonance $(\lambda=1.5452 \mu \mathrm{m})$ respectively, for $\varepsilon_{i}=0.165$. We observe that they are very similar to those of the lossless metal case [Figs. 1(b) and 1(e)]. Note that, even though the MDM plasmonic waveguide is lossy, the propagation length of its fundamental propagating mode is much longer than the device length here, which is set by the cavity length $a$. Thus, even though gain media are incorporated in only a selected device area, the device behaves as if the metal were lossless.
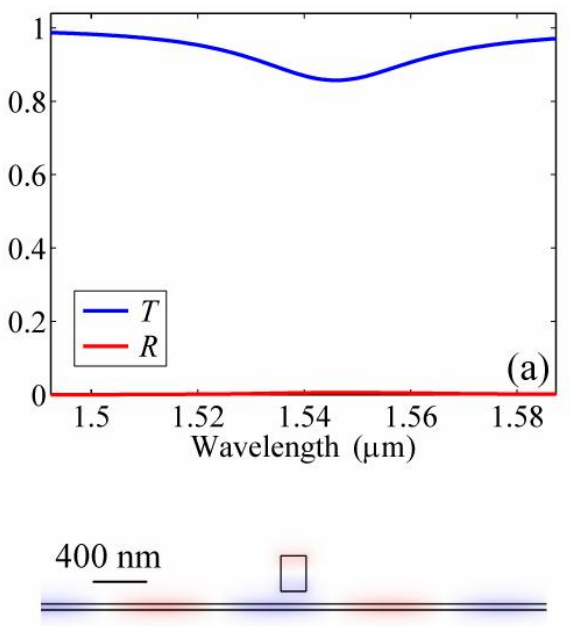

(c)
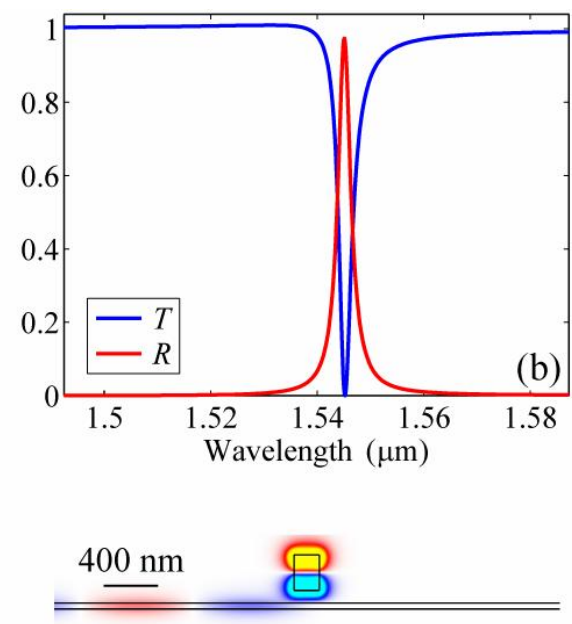

(d)

Fig. 3. (a) Same as Fig. 1(d), except that the cavity is filled with InGaAsP, and there is no pumping of InGaAsP ( $\varepsilon=11.38-i 0.1$ ). (b) Same as Fig. 3(a), except that there is pumping of $\operatorname{InGaAsP}(\varepsilon=11.38+i 0.165$ ). (c) Magnetic field profile of the device [shown in the inset of Fig. 1(d)] at $\lambda=1.5452 \mu \mathrm{m}$ in the absence of pumping ( $\varepsilon=11.38-i 0.1$ ). All other parameters are as in Fig. 3(a). (d) Magnetic field profile of the device [shown in the inset of Fig. 1(d)] at $\lambda=1.5452 \mu \mathrm{m}$ in the presence of pumping $(\varepsilon=11.38+i 0.165)$. All other parameters are as in Fig. 3(b).

Such a side-coupled structure in Fig.3 can act as a gain-assisted switch for MDM plasmonic waveguides. In the absence of 
pumping, the semiconductor material filling the cavity has $\varepsilon_{i}=-0.1$. And there is almost complete transmission of the incident optical wave through the plasmonic waveguide [Fig. 2(b)]. In Figs. 3(a) and 3(c) we show the transmission $T$ and reflection $R$ spectra, and the magnetic field profile at $\lambda=1.5452 \mu \mathrm{m}$ of the device, respectively, in the absence of pumping. We observe that an incident optical wave in the MDM plasmonic waveguide remains essentially undisturbed by the presence of the cavity. There is almost complete transmission of the incident optical wave through the MDM plasmonic waveguide $(T ; 0.86)$, while almost all the remaining portion of the incident optical power is absorbed in the cavity. In contrast, in the presence of pumping, the transmission decreases [Fig. 2(b)]. When the pumping is such that the material gain in the medium filling the cavity compensates the material loss in the metal $\left(\varepsilon_{i}=0.165\right)$, the incident optical wave is completely reflected at resonance [Figs. 3(b), 3(d)]. Thus, such a side-coupled structure acts as an extremely compact gain-assisted switch for MDM plasmonic waveguides, in which the on/off states correspond to the absence/presence of pumping.

As final remarks, the switching time in such a device will be limited by the carrier lifetime which is on the order of $0.2 \mathrm{~ns}$ [18]. In addition, we estimate that the pumping power in the off state will be on the order of $50 \mu \mathrm{W}$, by considering the required carrier density for gain coefficient [18], and assuming a device thickness of half a wavelength. Moreover, the

device proposed here may also function a plasmonic laser when the gain coefficient $g$ reaches above $2.16 \times 10^{3} \mathrm{~cm}^{-1}$. At such a gain, the cavity loss, including both the material loss and coupling to the waveguide, is compensated by the gain.

\section{ACKNOWLEDGMENTS}

This research was supported by DARPA/MARCO under the Interconnect Focus Center and by AFOSR grant FA 9550-04-1-0437.

\section{REFERENCES}

1. W. L. Barnes, A. Dereux, and T. W. Ebbesen, Nature 424, 824 (2003).

2. D. J. Bergman and M. I. Stockman, Phys. Rev. Lett. 90, 027402 (2003).

3. N. M. Lawandy, Appl. Phys. Lett. 85, 5040 (2004).

4. J. Seidel, S. Grafstrom, and L. Eng, Phys. Rev. Lett. 94, 177401 (2005).

5. M. A. Noginov, G. Zhu, M. Bahoura, J. Adegoke, C. E. Small, B. A. Ritzo, V. P. Drachev, and V. M. Shalaev, Opt. Lett. 31 , 3022 (2006).

6. M. P. Nezhad, K. Tetz, and Y. Fainman, Opt. Express 12, 4072 (2004).

7. S. A. Maier, Opt. Commun. 258, 295 (2006).

8. D. S. Citrin, Opt. Lett. 31, 98 (2006).

9. S. A. Ramakrishna and J. B. Pendry, Phys. Rev. B 67, 201101 (2003).

10. A. A. Govyadinov and V. A. Podolskiy, Phys. Rev. Lett. 97, 223902 (2006).

11. S. Xiao, L. Liu, and M. Qiu, Opt. Express 14, 2932 (2006).

12. A. Hosseini and Y. Massoud, Appl. Phys. Lett. 90, 181102 (2007).

13. S. D. Wu and E. N. Glytsis, J. Opt. Soc. Am. A 19, 2018 (2002).

14. G. Veronis, R. W. Dutton, and S. Fan, Opt. Lett. 29, 2288 (2004).

15. E. D. Palik, Handbook of Optical Constants of Solids (Academic, New York, 1985).

16. J. Jin, The Finite Element Method in Electromagnetics (Wiley, New York, 2002).

17. H. A. Haus and Y. Lai, IEEE J. Quantum Electron. 28, 205 (1992).

18. T. Saitoh and T. Mukai, IEEE J. Quantum Electron. QE-23, 1010 (1987).

19. N. Kirstaedter, O. G. Schmidt, N. N. Ledentsov, D. Bimberg, V. M. Ustinov, A. Y. Egorov, A. E. Zhukov, M. V. Maximov, P. S. Kopev, and Z. I. Alferov, Appl. Phys. Lett. 69, 1226 (1996). 